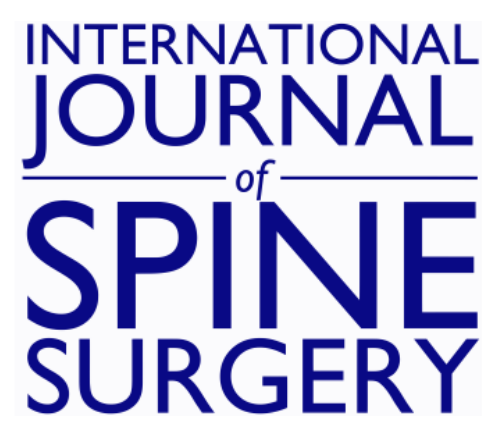

\title{
Elastic Image Fusion Software to Coregister Preoperatively Planned Pedicle Screws With Intraoperative Computed Tomography Data for Image-Guided Spinal Surgery
}

Franziska A. Schmidt, Mary Mullally, Martin Lohmann, Patrick Hiepe, Sertac Kirnaz, Swathi Chidambaram, Christoph Wipplinger and Roger Härtl

Int J Spine Surg 2021, 15 (2) 295-301

doi: https://doi.org/10.14444/8039

http://ijssurgery.com/content/15/2/295

This information is current as of April 25, 2023.

Email Alerts Receive free email-alerts when new articles cite this article. Sign up at: http://ijssurgery.com/alerts 


\title{
Elastic Image Fusion Software to Coregister Preoperatively Planned Pedicle Screws With Intraoperative Computed Tomography Data for Image-Guided Spinal Surgery
}

\author{
FRANZISKA A. SCHMIDT, MD, ${ }^{1}$ MARY MULLALLY, BS, ${ }^{2}$ MARTIN LOHMANN, MS, ${ }^{2}$

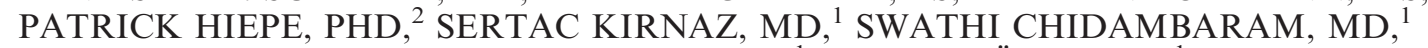 \\ CHRISTOPH WIPPLINGER, MD, ${ }^{1}$ ROGER HÄRTL, MD ${ }^{1}$ \\ ${ }^{I}$ Weill Cornell Brain and Spine Center, Department of Neurological Surgery, Weill Cornell Medical College, New York Presbyterian Hospital, \\ New York, New York, ${ }^{2}$ BrainLAB AG, Munich, Germany
}

\begin{abstract}
Background: For complex spinal cases, especially when robotic guidance is used, preoperative planning of pedicle screws can be helpful. Transfer of these preoperatively planned pedicle screws to intraoperative 3dimensional imaging is challenging because of changes in anatomic alignment between preoperative supine and intraoperative prone imaging, especially when multiple levels are involved. In the spine, where each individual vertebra is subject to independent movement from adjacent level, rigid image fusion is confined to a single vertebra and can display fusion inaccuracies on adjacent levels. A novel elastic fusion algorithm is introduced to overcome these disadvantages. This study aimed to investigate image registration accuracy of preoperatively planned pedicle screws with an elastic fusion algorithm vs. rigid fusion for intraoperative placement with imageguided surgery.
\end{abstract}

Methods: A total of 12 patients, were selected depending on the availability of a preoperative spinal computed tomography (CT) and an intraoperative AIRO CT scan (BrainLAB AG, Munich, Germany) of the same spinal region. To verify accuracy differences between rigid fusion and elastic fusion 76 bilateral screw trajectories were virtually defined in the preoperative CT image, and they were transferred via either rigid fusion or elastic fusion to the intraoperative CT scan. Accuracy of the transferred screws in the rigid and elastic fusion group was determined by measuring pedicle breaches on the intraoperative CT.

Results: In the rigid fusion group $1.3 \%$ of screws showed a breach of less than $2 \mathrm{~mm}, 9.2 \%$ showed breaches between 2 and $4 \mathrm{~mm}$, and $18.4 \%$ of the screws showed an error above $4 \mathrm{~mm}$. The elastic fusion group showed no breaches and provided high accuracy between preoperative and intraoperative screw placement.

Conclusion: Elastic fusion provides high registration accuracy and represents a considerable step towards efficiency and safety in CT-based image-guided surgery.

Level of Evidence: 3.

New Technology

Keywords: screw placement, 3D-NAV, pedicle screw, AIRO, intraoperative CT, navigated spine surgery, image-guided surgery

\section{INTRODUCTION}

For complex spine cases with challenging anatomy it may be beneficial to preplan screws on a preoperative computed tomography (CT) scan and then transfer by coregistering these screws to the intraoperative CT scan for navigated or robotic placement of screws. This can accelerate the intraoperative workflow, because the surgeon does not have to plan or assess the pedicle screw position in the OR and can rely on a preoperative plan. Especially for robotic navigation systems preoper- ative planning is used routinely. Rigid image fusion is linked to static anatomic structures. Due to different patient positioning in the preoperative (supine) and the intraoperative (prone) stages, the accuracy of this software can be diminished. Therefore, the user has to perform several image fusions for the different segments of the spine to gain an accurate coregistration of the preoperatively planned screws. This is time-consuming, and therefore, not applicable for most surgeries. BrainLAB has developed an elastic fusion software, which can fuse 2 data sets accurately over the whole spine and 
thereby compensate for different patient positioning in the preoperative and the intraoperative setting. The software is capable of fusing the preoperative data set to the intraoperative data set and thereby transforming the curvature so that it matches over all the vertebrae along the spine with the patient position of the intraoperative data set. Until now, this coregistration was performed with rigid fusion software, which can fuse 2 data sets in a predefined region of interest (ROI). The aim of the study is to compare the performance of the rigid fusion software to the elastic fusion software. With this comparison, we want to evaluate if elastic fusion would allow for an accurate, reliable, and efficient coregistration and transfer of preoperatively planned screws to the intraoperative setting, to be used for image-guided surgery.

\section{METHODS}

\section{Data Collection}

A retrospective single-center study of 12 (3 female, 9 male) patients who underwent instrumented fusion was performed (Weill Cornell Medical College Institutional Review Board/Ethics Committee approval protocol number 0806009851).

The indication for surgery was spondylolisthesis and spinal canal stenosis. Patients were positioned prone onto a Jackson table. The median age of our patients was 71 years (range, 36-84 years) and the average body mass index was $27 \mathrm{~kg} / \mathrm{m}^{2}$.

All patients had preoperative CT scans. Screw positioning was planned on preoperative CT scans. Screws were then coregistered with the 2 different 3D navigation software programs. Each screw was coregistered with rigid fusion (Elements Image Fusion 3.0) and with elastic fusion (Elements Curvature Correction Spine 3.0). Please note that we were using 2 CT scans (preoperative and intraoperative) in order to have the same ROI from the same patient. Between 2 and 4 levels have been planned virtually on the thoracolumbar spine. The study was conducted with virtually planned screws in order to understand the clinical difference of rigid fusion (Elements Image Fusion 3.0) and elastic fusion (Elements Curvature Correction Spine 3.0).

\section{Steps of Approach}

We collected preoperative CT scans and intraoperative CT scans from 12 patients who underwent spinal surgery unrelated to this study
1. Pedicle screws were preplanned manually in the preoperative CT scan

2. The preoperative CT scans (including screws) were fused to the intraoperative CT scan:

a. With rigid fusion

b. With elastic fusion

3. Screw accuracy of the planned and then virtually transferred pedicle screws in the intraoperative CT scan was analyzed with DICOM Viewer

4. To quantify the measured breach, we used the Gertzbein ${ }^{1}$ criteria:

a. In the rigid fusion plan

b. In the elastic fusion plan

The basic difference between elastic and rigid fusion is that the methods used to determine the elastic fusion encompass the methods used to calculate the rigid fusion for each vertebra. Similarly to rigid fusion, each region of interest in elastic fusion is considered separately but will also take the results of the adjacent previous ROIs into account.

The fusion calculation acquired for a certain vertebra assists as contribution for the following fusion of the adjacent vertebra. This method gives a number of vertebra-specific rigid fusions which are necessary to define a nonrigid deformation field in elastic fusion. ${ }^{2,3}$

\section{Radiologic Evaluation}

We performed a blinded evaluation of all coregistered CT scans. The 3-dimensional (3D) navigation system was used to determine the accuracy of the virtually planned pedicle screws. The images have been constructed in the axial, sagittal, and coronal planes. The CT scans were evaluated using the grading system described by Gertzbein et al ${ }^{1,4}$ previously: grade 0: perfect screw. The screw is within the cortical margins. Grade 1: for perforation distance between 0 and $2 \mathrm{~mm}$. Grade 2: perforation distance between 2 and $4 \mathrm{~mm}$. Grade 3: perforation distance greater than $4 \mathrm{~mm}$. The accuracy of each individual screw was graded in rigid fusion and elastic fusion (Figure 1).

Lateral perforation distance is the distance in the axial plane between the lateral cortex of the pedicle and the outer lateral margin of the screw threads. Medial perforation distance is the distance in the axial plane between the medial cortex and the medial outer margin of the screw threads. Coronal perforation distance is the distance of the pedicle to the outer margin of the screw threads. 

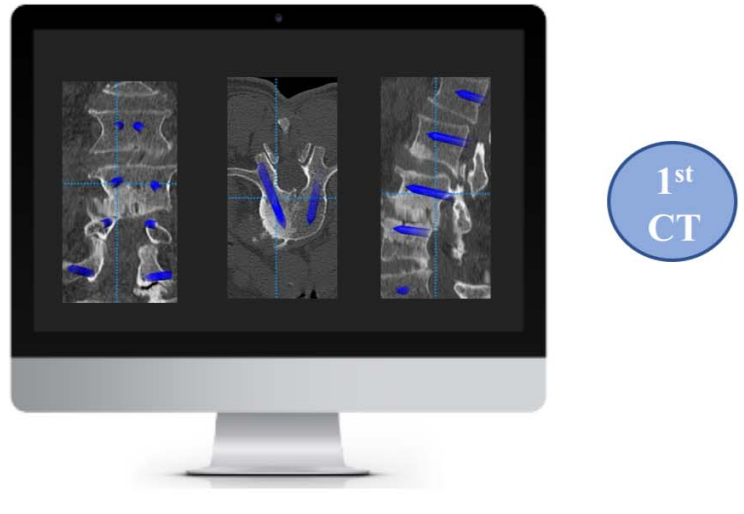

preoperative

intraoperative

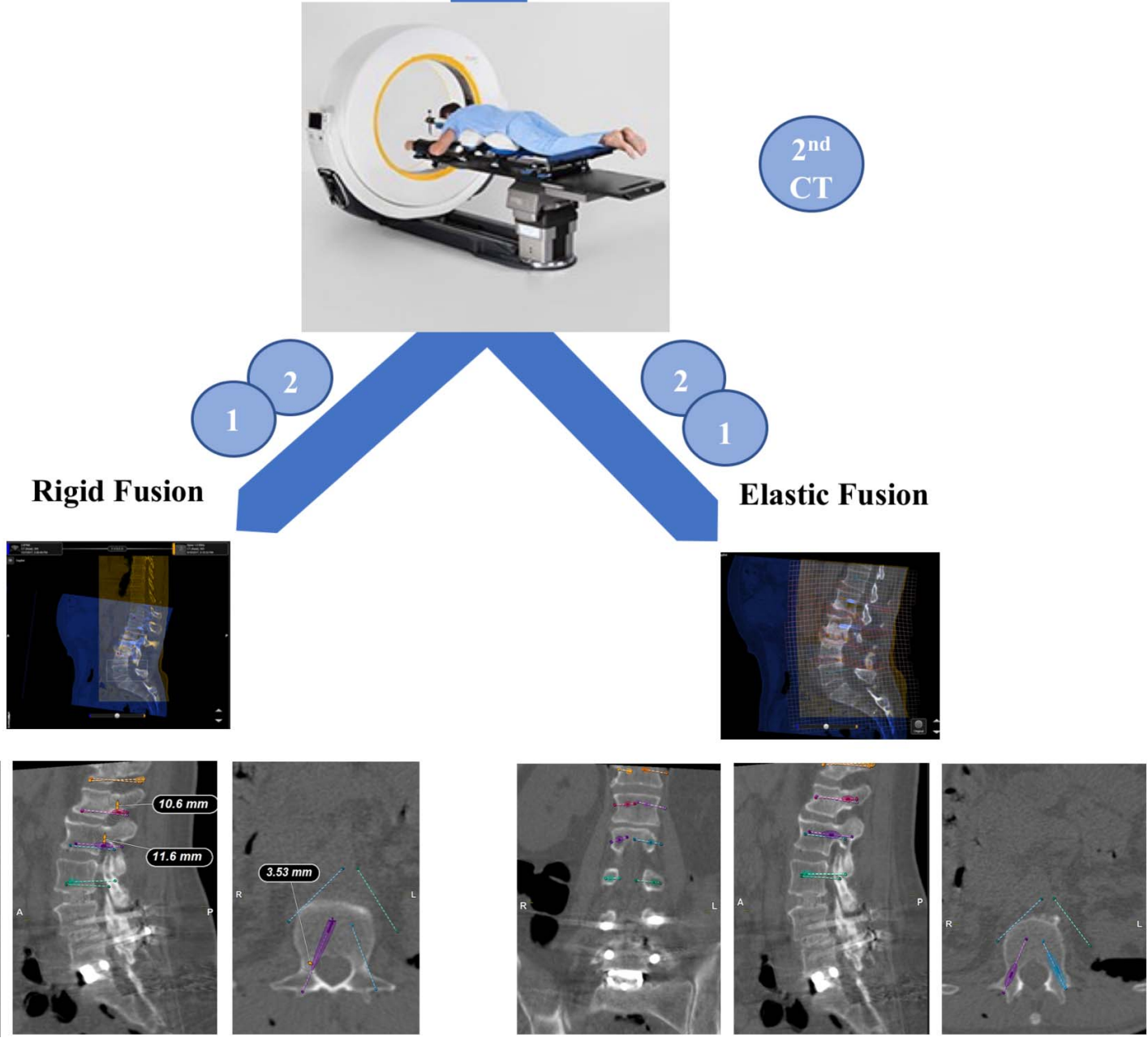

Figure 1. Flowchart describing our technique in comparing rigid and elastic fusion. First, screws are planned using a preoperative computed tomography (CT) scan, then the planned screws are transferred to the intraoperative AIRO CT scan with rigid fusion and with elastic fusion. There is a $71 \%$ accuracy rate of preoperative planned screws in rigid fusion and a $100 \%$ accuracy rate of preoperative planned screws in elastic fusion.

\section{RESULTS}

Between 2 and 4 levels were evaluated ( 2 levels: $\mathrm{n}=1$ [8\%], 3 levels: $\mathrm{n}=8$ [67\%], 4 levels: $\mathrm{n}=3$ [25\%]). Screws were placed in thoracic and lumbar spine. The 76 pedicle screws in our 12 patients were visualized on
CT scans with the DICOM viewer to enable an accuracy assessment.

\section{Rigid Fusion}

Based on the grading system described by Gertzbein et al, ${ }^{1}$ in the rigid fusion group 54 screws 
Elastic Image Fusion Software and Intraoperative CT Data for Spine Surgery

Table. Type and direction of pedicle breaches in rigid fusion (note: a breach could have more than 1 direction).

\begin{tabular}{|c|c|c|c|c|c|c|c|c|}
\hline Breach & Axial Breach & Coronal Breach & Sagittal Breach & Medial & Lateral & Cranial & Caudal & Total, No. (\%) \\
\hline Grade 0 & - & - & - & - & - & - & - & - \\
\hline Grade 1 & 1 & 2 & 2 & 1 & - & - & 2 & $1(1.3)$ \\
\hline Grade 2 & 2 & 9 & 6 & 2 & - & 3 & 4 & $7(9.2)$ \\
\hline Grade 3 & - & 6 & 13 & - & 1 & 3 & 10 & 14 (18.4) \\
\hline
\end{tabular}

(71\%) were grade $0,1(1.3 \%)$ was grade $1,7(9.2 \%)$ were grade 2, and $14(18.4 \%)$ were grade 3 breaches. We differentiated between medial and lateral as well as cranial and caudal breaches. Screw perforation occurred medially 3 times and laterally once. A total of 16 screws had a caudal breach and 6 a cranial breach (Table).

\section{Elastic Fusion}

In the elastic fusion group no breach occurred in the sagittal, coronal, or axial plane. Each of the 76 screws reached grade 0 (Table).

\section{DISCUSSION}

Our quantified analysis of the screw positions showed that $29 \%$ of the rigid fusion patients experienced suboptimal screw trajectories in the intraoperative CT scan. Therefore, these screw trajectories could not be used for image-guided/ robotic surgery and would have to be replanned in the operating room (OR), leading to additional OR time. This screw misplacement rate did not appear in line with the numbers quoted in previous reports. ${ }^{5-8}$ However, elastic fusion showed an accuracy rate of $100 \%$, with none of the screws demonstrating a significant breach.

In this study, we demonstrated that rigid fusion can only be applied in a local ROI. Thereby only preplanned screws within this ROI are coregistered accurately, whereas screws outside of this ROI can be coregistered inaccurately (Figures 2 and 3). The resulting inaccuracy is a function of the difference of spinal curvature between the 2 data sets. Curvature between 2 data sets can differ mainly because of positioning (supine position in the preoperative imaging and prone in intraoperative imaging). If the curvature does not differ greatly, the surgeon might be able to coregister screws outside the defined ROI. However, if the curvature does differ, this is no longer possible, and multiple ROIs have to be defined. Furthermore, multiple fusions have to be performed to transfer all screws. This is timeconsuming and most likely not feasible within clinical practice. To measure this effect and to analyze the benefit of the elastic fusion method, we fused several pairs of data sets with the rigid fusion using only 1 ROI. Subsequently, the breaches of the screws after coregistration were measured. We subsequently found that breaches of the screws are a function of the difference in the curvatures between the preoperative and intraoperative data sets. This further highlights the utility of the elastic fusion method for preoperative screw planning for navigation.

Elastic fusion provides fusion of preoperative magnetic resonance imaging or CT scans and intraoperative CT image data with a median error below $1.34 \mathrm{~mm} .{ }^{9,10}$ In contrast to rigid fusion, elastic fusion automatically calculates individual rigid fusions for each vertebra and from these results interpolates a single 3D deformation field that simultaneously matches all vertebrae in the fused images. ${ }^{2,3}$ This leads to a level-by-level coregistration. ${ }^{11}$

The real benefit of elastic fusion hinges on its efficiency. Preoperative position planning of the screws is feasible and more attractive especially in longer constructs (Figure 4). In our experience, elastic fusion with the intraoperative CT was efficient and easy to implement. The fact that screws could be planned preoperatively supports shorter OR time and improved workflow. The future in navigation will continue to evolve over the coming years. Software improvements, such as the one described here, will be instrumental in advancing the field of spinal navigation.

\section{LIMITATION}

This paper outlines an initial, single-institution evaluation of virtually planned screws, relying on quantitative analyses of retrospective patient data. The results are based on a single output value, breach accuracy. A comprehensive, prospective cohort study with more patients to determine screw accuracy will be needed. 

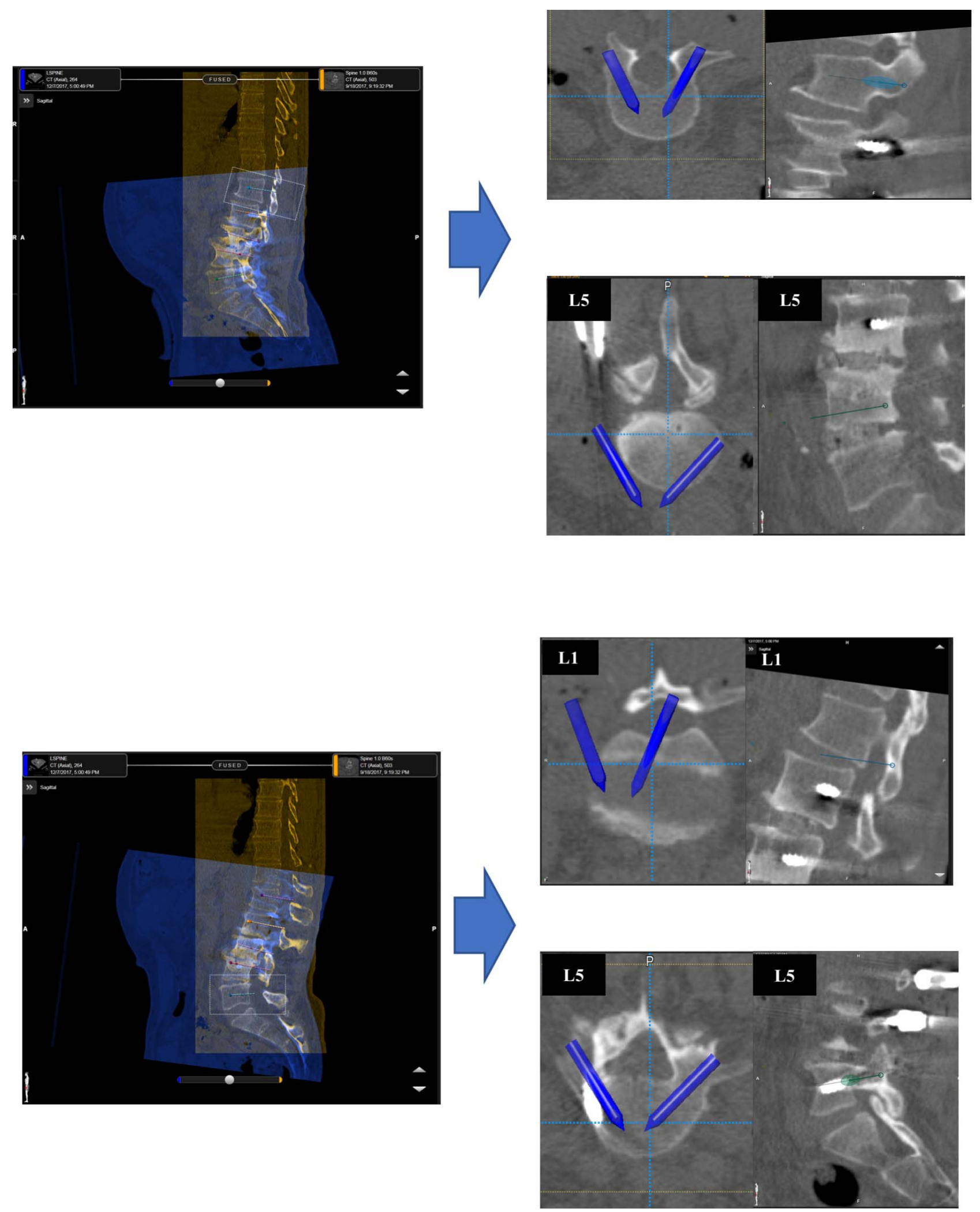

Figure 2. Figure showing coregistration of planned screws to intraoperative computed tomography (CT) scan for navigation with rigid fusion. Accuracy is not shown in every level. 

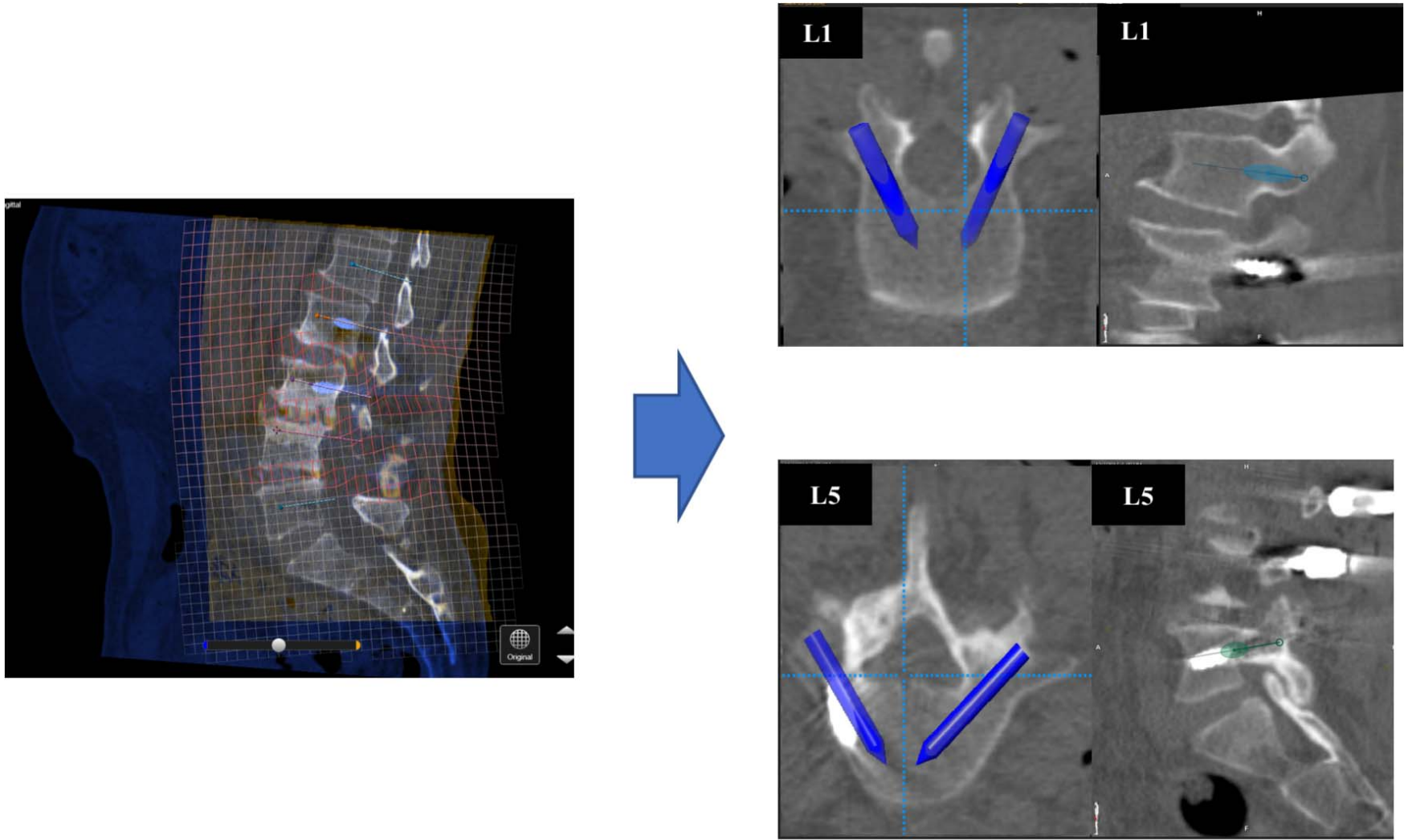

Figure 3. Figure showing coregistration of planned screws to intraoperative computed tomography (CT) scan for navigation with elastic fusion. Accuracy is shown throughout all levels.
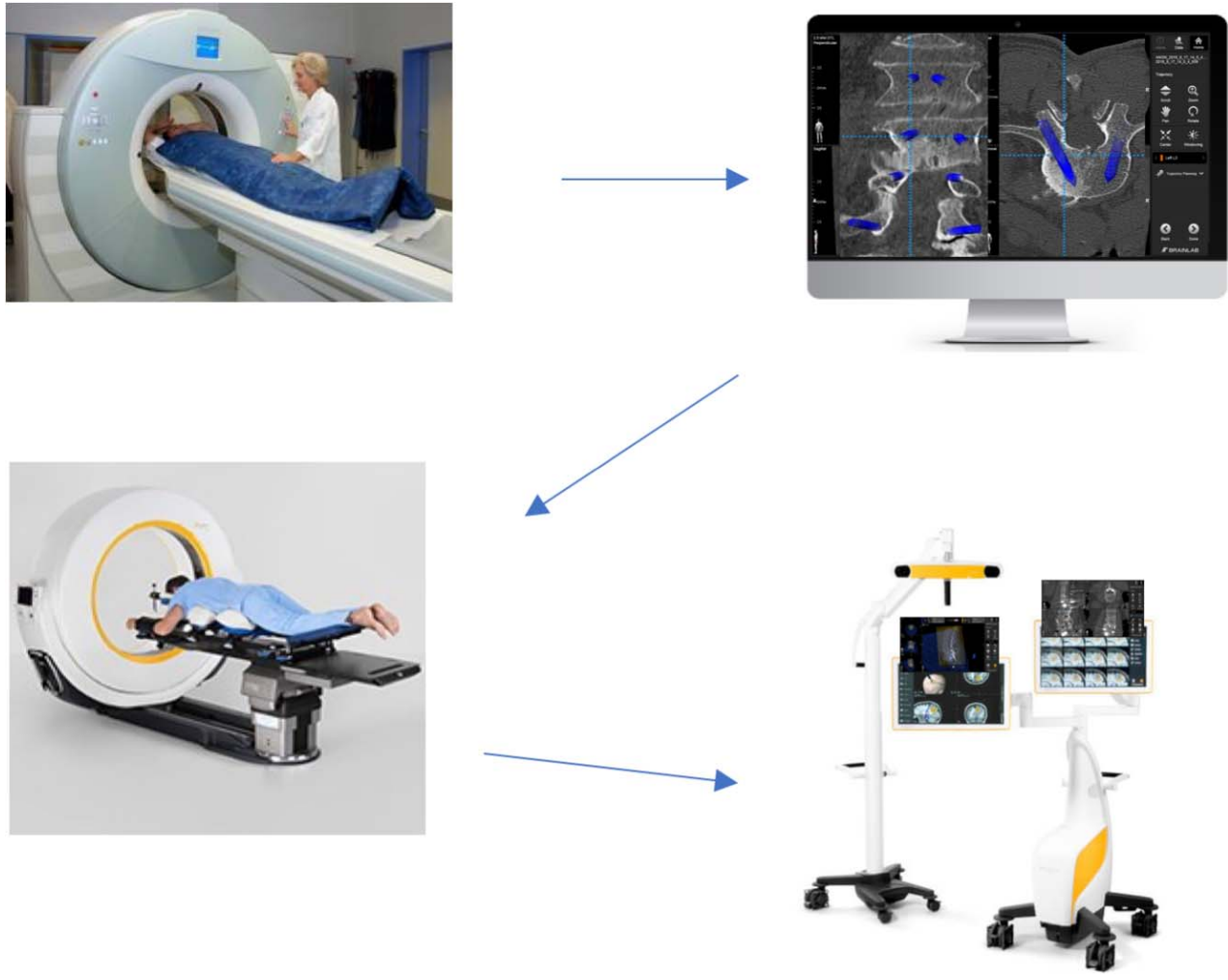

Figure 4. Flowchart illustrating the workflow of preoperative screw position planning with elastic fusion. Step 1: preoperative computed tomography (CT) acquisition in supine. Step 2: preoperative screw planning on CT on office computer. Step 3: intraoperative AIRO CT in prone. Step 4: coregistration/transfer of planned screws to intraoperative AIRO CT scan for navigation. 


\section{CONCLUSION}

The elastic fusion software enables reliable, accurate, and fast coregistration of preplanned screws. In our series of virtually planned screws it shows the potential to increase the efficiency and accuracy in the OR and make preoperative planning more beneficial for image-guided surgery and robotic surgery.

\section{REFERENCES}

1. Gertzbein SD, Robbins SE. Accuracy of pedicular screw placement in vivo. Spine. 1990;15(1):11-14.

2. Bamba Y, Nonaka M, Nakajima S, Yamasaki M. Threedimensional reconstructed computed tomography-magnetic resonance fusion image-based preoperative planning for surgical procedures for spinal lipoma or tethered spinal cord after myelomeningocele repair. Neurologia medico-chirurgica. 2011;51(5):397-402.

3. So TY, Lam YL, Mak KL. Computer-assisted navigation in bone tumor surgery: seamless workflow model and evolution of technique. Clin Orthop Relat Res. 2010;468(11):2985-2991.

4. Puvanesarajah V, Liauw JA, Lo SF, Lina IA, Witham TF. Techniques and accuracy of thoracolumbar pedicle screw placement. World J Orthop. 2014;5(2):112-123.

5. Gelalis ID, Paschos NK, Pakos EE, et al. Accuracy of pedicle screw placement: a systematic review of prospective in vivo studies comparing free hand, fluoroscopy guidance and navigation techniques. Eur Spine J. 2012;21(2):247-255.

6. Waschke A, Walter J, Duenisch P, Reichart R, Kalff R, Ewald C. CT-navigation versus fluoroscopy-guided placement of pedicle screws at the thoracolumbar spine: single center experience of 4,500 screws. Eur Spine J. 2013;22(3):654-660.

7. Tian W, Liu Y, Zheng S, Lv Y. Accuracy of lower cervical pedicle screw placement with assistance of distinct navigation systems: a human cadaveric study. Eur Spine J. 2013;22(1):148155.

8. Rivkin MA, Yocom SS. Thoracolumbar instrumentation with CT-guided navigation (O-arm) in 270 consecutive patients: accuracy rates and lessons learned. Neurosurg Focus. 2014;36(3):E7. doi: 10.3171/2014.1.FOCUS13499

9. Rana M, Essig H, Eckardt AM, et al. Advances and innovations in computer-assisted head and neck oncologic surgery. Journal of Craniofac Surg. 2012;23(1):272-278.

10. Otake Y, Wang AS, Uneri A, et al. 3D-2D registration in mobile radiographs: algorithm development and preliminary clinical evaluation. Physics Med Biol. 2015;60(5):2075-2090.

11. Rashad A, Heiland M, Hiepe P, et al. Evaluation of a novel elastic registration algorithm for spinal imaging data: a pilot clinical study. Int J Med Robot. 2019:e1991. doi: 10.1002/ res. 1991

12. Shin BJ, James AR, Njoku IU, Hartl R. Pedicle screw navigation: a systematic review and meta-analysis of perforation risk for computer-navigated versus freehand insertion. $J$ Neurosurg Spine. 2012;17(2):113-122.

13. Kosmopoulos V, Schizas C. Pedicle screw placement accuracy: a meta-analysis. Spine. 2007;32(3):E111-E120.

14. Luther N, Iorgulescu JB, Geannette C, et al. Comparison of navigated versus non-navigated pedicle screw placement in 260 patients and 1434 screws: screw accuracy, screw size, and the complexity of surgery. J Spinal Disord Techniques. 2015;28(5):E298-E303.

15. Farah K, Coudert P, Graillon T, et al. Prospective comparative study in spine surgery between O-Arm and Airo Systems: efficacy and radiation exposure. World Neurosurg. 2018;118:e175-e184.

16. Hecht N, Kamphuis M, Czabanka M, et al. Accuracy and workflow of navigated spinal instrumentation with the mobile AIRO((R)) CT scanner. Eur Spine J. 2016;25(3):716723.

17. Zausinger S, Scheder B, Uhl E, Heigl T, Morhard D, Tonn JC. Intraoperative computed tomography with integrated navigation system in spinal stabilizations. Spine. 2009;34(26):2919-2926.

18. Navarro-Ramirez R, Lang G, Lian X, et al. Total navigation in spine surgery; a concise guide to eliminate fluoroscopy using a portable intraoperative computed tomography 3-dimensional navigation system. World Neurosurg. 2017;100:325-335.

19. Hartl R, Lam KS, Wang J, Korge A, Kandziora F, Audige L. Worldwide survey on the use of navigation in spine surgery. World Neurosurg. 2013;79(1):162-172.

Disclosures and COI: R.H. has received funding from BrainLAB and Nuvasive; M.M., M.L., and P.H. are employees of BrainLAB. The other authors received no funding for this study and report no conflicts of interest.

Corresponding Author: Roger Härtl, MD, Weill Cornell Brain and Spine Center, Weill Cornell Medicine, Department of Neurological Surgery, 525 East 68th Street, Box 99, New York, NY 10065. Phone: (212) 746-2152; Email: roh9005@med. cornell.edu.

Published 16 April 2021

This manuscript is generously published free of charge by ISASS, the International Society for the Advancement of Spine Surgery. Copyright $\odot 2021$ ISASS. To see more or order reprints or permissions, see http://ijssurgery.com. 